University of Montana

ScholarWorks at University of Montana

$4-1-1980$

\title{
Studies of the Reorientational Relaxation of Pyridine in Water by Depolarized Rayleigh Light Scattering
}

\author{
C. H. Wang \\ University of Utah \\ Scott L. Whittenburg \\ University of Montana - Missoula, scott.whittenburg@umontana.edu \\ P. A. Lund \\ University of Copenhagen \\ D. H. Christensen \\ University of Utah
}

Follow this and additional works at: https://scholarworks.umt.edu/chem_pubs

Part of the Biochemistry Commons, Chemistry Commons, and the Physics Commons Let us know how access to this document benefits you.

\section{Recommended Citation}

Wang, C. H.; Whittenburg, Scott L.; Lund, P. A.; and Christensen, D. H., "Studies of the Reorientational Relaxation of Pyridine in Water by Depolarized Rayleigh Light Scattering" (1980). Chemistry and Biochemistry Faculty Publications. 72.

https://scholarworks.umt.edu/chem_pubs/72

This Article is brought to you for free and open access by the Chemistry and Biochemistry at ScholarWorks at University of Montana. It has been accepted for inclusion in Chemistry and Biochemistry Faculty Publications by an authorized administrator of ScholarWorks at University of Montana. For more information, please contact scholarworks@mso.umt.edu. 


\title{
Studies of the reorientational relaxation of pyridine in water by depolarized Rayleigh light scattering
}

\author{
C. H. Wang and S. L. Whittenburga) \\ Department of Chemistry, University of Utah, Salt Lake City, Utah 84112
}

P.-A. Lund and D. H. Christensen

Department of Chemical Physics, The H. C. Orsted Institute, University of Copenhagen, KD-2100 Copenhagen, Denmark

(Received 16 July 1979; accepted 14 August 1979)

\begin{abstract}
The depolarized Rayleigh spectra of aqueous solutions of pyridine have been studied using a high-finesse Fabry-Perot interferometer as a function of temperature and concentration. The Rayleigh relaxation times are found to have a complex concentration and viscosity dependence. The classical Stokes-Einstein-Debye equation for molecular reorientation breaks down in this system. The Rayleigh relaxation time of pyridine molecules is not determined by the macroscopic shear viscosity of the solution. The specific interaction due to the formation of hydrogen bonds between pyridine and water molecules plays a very important role in affecting the relaxation time. At a fixed temperature the plot of $\pi_{\text {ray }} / \eta$ versus pyridine concentration shows two maxima at low and high pyridine concentrations. The low concentration maximum is due to the incorporation of pyridine molecules in the water network structure and the high concentration maximum is associated with the formation of individual pyridine-water complexes. The activation energy for the reorientation of pyridine molecules depends on the pyridine concentration. At low pyridine concentration the activation energy corresponds well to the $\mathrm{N} . . \mathrm{H}-\mathrm{O}$ hydrogen bonding energy. Above $70 \%$ volume the activation energy decreases with increasing pyridine concentration, and above this concentration range the reorientational relaxation time becomes less structure controlled.
\end{abstract}

\section{INTRODUCTION}

The reorientational motion of simple molecules in a dense molecular fluid is an important area in theoretical and experimental research. A complete description of the reorientational motion requires a knowledge of single particle dynamics as well as the angular correlation between the reorientations of different molecules. Light scattering provides a way of measuring both contributions. Raman scattering measures the average rate of reorientation of the symmetry axes of individual molecules while depolarized Rayleigh light scattering probes the single particle rotation as well as the correlated reorientation of molecular pairs. The contribution of both the single particle reorientation and the pair angular correlation effect on the depolarized Rayleigh relaxation time of an anisotropic solute in a solution as a function of concentration in an optically isotropic solvent can be determined with the theory due to Keyes and Kivelson. ${ }^{1}$ In the limit of infinite dilution the depolarized Rayleigh measurement probes single particle reorientation. ${ }^{2}$ Thus, depolarized Rayleigh light scattering provides a valuable method for studying single particle rotation and orientational pair correlation (OPC). ${ }^{3,4}$

The single particle reorientation times $\tau_{s}$ for a large variety of molecular liquids consisting of small molecules have been found to be a linear function of shear viscosity $(\eta)$ for a solution at a fixed temperature $(T)$. Experimentally, $\tau_{s}$ is known to be given by

$$
\tau_{s}=(V \eta / k T) \kappa+\tau_{0},
$$

where $V$ is the effective volume of the reorienting mole-

\footnotetext{
a) Present address: Department of Chemistry, University of New Orleans, New Orleans, LA 70122.
}

cule, and $k$ is the Boltzmann constant. $\tau_{0}$ is the reorientational relaxation time obtained by extrapolating $\tau_{s}$ to a zero $\eta / T$ value. The origin of $\tau_{0}$ does not have a clear theoretical understanding at present. In the case that the reorienting molecule becomes much larger than the size of molecules which form the solvent, $\tau_{0}$ becomes negligible and $\tau_{s}$ is effectively given by the first term. The parameter $k$ is a dimensionless quantity which measures the interaction of the rotational motion of the reorienting molecules with the translational motion of the fluid. In many cases it is found that $\kappa$ is independent of temperature and viscosity but depends upon the specific solvent-solute interaction. ${ }^{5-7}$ The original StokesEinstein-Debye formula was derived in the hydrodynamic limit with the so-called stick boundary condition corresponding to $\kappa=1.8,9$ Experience has shown, however, that $\kappa<1$ is necessary to account for the experimental result of most nonassociating molecular liquids. ${ }^{4,10-12}$ Recently, Hu and $\mathrm{Zwanzig}^{13}$ have analyzed the reorientation of a spheroidal and an ellipsoidal molecule in a fluid where stick boundary conditions do not hold. Their proposed use of "slip" boundary conditions yields $\kappa<1$.

The linear relationship between $\tau_{s}$ and $\eta$ at a fixed fluid temperature is known to fail when the viscosity is high. ${ }^{14,15}$ One also expects the linear relationship to fail when a specific solute-solvent interaction such as hydrogen-bond formation is present. For the hydrogenbonding solvent the hydrodynamic picture which leads to Eq. (1), with $\tau_{0}=0$, is probably rather poor, as the formation of molecular associates leads to a complex liquid structure with the structural relaxational effect becoming an important factor in influencing the reorientational dynamics of the solute molecule. The effect of structural relaxation on the molecular reorientation has 


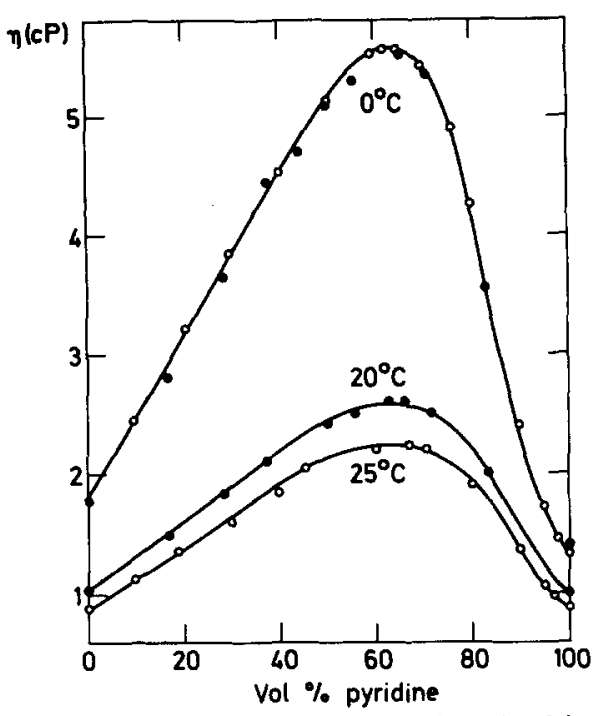

FIG. 1. Viscosity of pyridine dissolved in water (as a function of py ridine concentration) at 0 and $20^{\circ} \mathrm{C}$ (solid circles). Additional data of 0 and $35^{\circ} \mathrm{C}$ (empty circles) are taken from Ref. 22 .

not been included in the hydrodynamic theory. Structural relaxation is expected to be a very important factor in determining the reorientational time in systems in which hydrogen-bonding interactions are important.

In the investigation of the viscosity effect on the reorientational relaxation time in a solution by depolarized Rayleigh scattering, it is important to take into account the fact that, as the concentration of the anisotropic molecule is changed, the viscosity of the solution is often altered, even if the viscosity of both the neat solvent and solute are the same. For systems in which the viscosity of the solution varies linearly with concentration, the reorientational relaxation time measured by the depolarized Rayleigh scattering technique $\left(\tau_{\mathrm{R} \text { ar }}\right)$ may be scaled with respect to the solution viscosity and accurate single particle reorientational relaxation times of pure liquids obtained by extrapolation. ${ }^{2,4}$ In the case of pyridine- $\mathrm{CCl}_{4}{ }^{16}$ and pyridine-cyclohexane ${ }^{17}$ systems in which the solute-solvent interaction gives rise to a nonlinear concentration-viscosity relation, we have found the viscosity-scaling technique for extracting the single particle reorientation time still remains valid.

In the pyridine- $\mathrm{CCl}_{4}$ or pyridine-cyclohexane system the solute-solvent interaction gives rise to only a $10 \%$ deviation from linearity in the concentration versus viscosity curve. In contrast, the viscosity of a $66 \%$ by volume pyridine-water solution is 2.6 times the viscosity of either neat solute or solvent. The effect of hydrogen bonding on molecular vibrations of pyridine has been studied using IR spectroscopy. ${ }^{18}$ Also, Raman scattering studies have shown that in the pyridine-water system, the specific hydrogen-bonding interaction leads to the formation of short-lived molecular complexes which give rise to significant concentration dependent linewidths and line shapes of the polarized and depolarized Raman spectra. ${ }^{19,20}$ Therefore, a depolarized Rayleigh light scattering study of the pyridine-water system will provide an excellent test of the viscosity scaling hypothesis as well as a measure of the importance of the nonhydrodynamic effect on the reorientational motion of the pyridine molecules due to hydrogen bonding. In this paper we shall describe results of depolarized Rayleigh scattering experiments on the pyridine-water system at various temperatures and concentrations, with particular emphases on those aspects associated with molecular reorientation and nonhydrodynamic interaction. Our basic conclusions on the pyridine-water system are that Eq. (1) is not obeyed and that the nonhydrodynamic interaction has an important effect on the reorientational relaxation of pyridine molecules. Preliminary results of this work were previously reported elsewhere. ${ }^{21}$

\section{EXPERIMENTAL}

The depolarized Rayleigh scattering of aqueous pyridine solutions have been obtained by the same technique and instrumentation as used for neat pyridine and pyridine$\mathrm{CCl}_{4}$ solutions. ${ }^{16}$ The aqueous solutions were prepared from spectraquality grade pyridine and redistilled water and were repeatedly filtered through $0.22 \mathrm{~mm}$ millipore filters to remove dust. Filtration proceeded very slowly (typical filtration time $\sim 12 \mathrm{~h}$ ). The final concentration of the samples were certified by measuring the index of refraction of the solutions before and after the light scattering experiment.

Viscosity data for water-pyridine mixtures at several temperatures are found in Ref. 22. The viscosities given for water-pyridine mixtures at $0^{\circ} \mathrm{C}$ were supplemented by a series of measurements made with a Cannon-Ubbelhode viscometer submerged in a waterethylene glycol bath. The viscosity-concentration curves at several temperatures are given in Fig. 1. Also the viscosity-concentration curve for aqueous pyridine solutions at $20^{\circ} \mathrm{C}$ were obtained using the same constant temperature bath as used for the light scattering experiments.

The experimental spectra were recorded on an $X-Y$ recorder and digitized. The free spectral range of the Fabry-Perot interferometer was $300 \mathrm{GHz}$. A typical pyridine-water spectrum together with a spectrum of pure water under the same experimental conditions is shown in Fig. 2. The contribution to the depolarized intensity from the water solvent is negligible. The digitized spectra were corrected for an instrumental width corresponding to a typical finesse of 60 and were

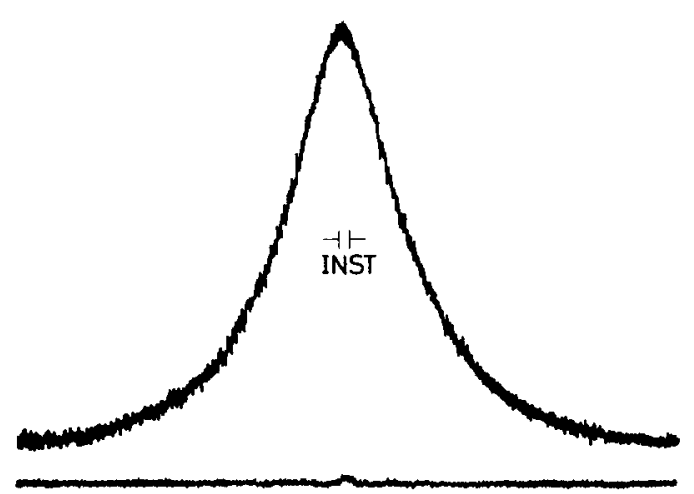

FIG. 2. Depolarized Rayleigh spectrum of a $77 \%$ solution of pyridine in water at $29^{\circ} \mathrm{C}$ together with the pure water spectrum recorded under the same conditions. 
fit to overlapping Lorentzian functions plus a constant background.

\section{RESULTS AND DISCUSSION}

The depolarized Rayleigh spectrum of a liquid solution is a measure of the fluctuations in the macroscopic polarizability density due to reorientations of both solute and solvent molecules. For optically isotropic solvent molecules the depolarized spectrum reflects only the reorientation of the anisotropic solute molecules. Water as a solvent is not optically isotropic and therefore contributes to the total depolarized scattering intensity in the pyridine-water solution. Nevertheless, the reorientation of the pyridine molecules can still be studied, due to the fact that the contribution from fluctuations in the polarizability density of pyridine is much greater than that of water. Shown in Fig. 2 are depolarized Rayleigh spectra of the $77 \%$ pyridine-water solution and neat water under identical experimental conditions. One notes that the intensity of the light scattered from neat water is negligibly small compared to the intensity from pyridine. Thus, light scattered from solutions of pyridine in water effectively reflects the reorientation only of the pyridine molecules.

The rotational relaxation time of an asymmetric top molecule represents a weighted average of the rate of reorientation about the three principal axes. While pyridine is strictly an asymmetric top it is isoelectronic with benzene with a polarizability tensor nearly equal to that of a symmetric top molecule. ${ }^{23}$ Previous depolarized Rayleigh scattering studies on pyridine have shown good agreement with earlier experimental results for benzene. ${ }^{16}$ Thus, depolarized Rayleigh scattering of liquid pyridine can be approximated as scattering from a liquid of symmetric top molecules.

The Rayleigh relaxation times $\tau_{\mathrm{Ray}}$ are obtained from the half-width at half-height ( $\Gamma$ in $\mathrm{Hz}$ ) of the experimental spectra according to

$$
\tau_{\mathrm{Ray}}=(2 \pi \Gamma)^{-1} \text {. }
$$

The experimentally determined $\tau_{\mathrm{Ray}}$ at $0^{\circ}$ and $20^{\circ} \mathrm{C}$ are given in Table $I$ for various concentrations along with the measured values of $\eta / T$, where $\eta$ is the shear viscosity of the solution and $T$ is the absolute temperature. It should be pointed out that our $\tau_{R_{2} y}$ values are consistently longer than that obtained by Schindler and Posch using a double-grating monochromator to study the depolarized Rayleigh spectra of the pyridine-water system. ${ }^{19}$ As stressed in several of our previous papers, ${ }^{2,4,24}$ to analyze the reorientational relaxation time by depolarized Rayleigh scattering, great care must be taken if a conventional double-grating monochromator is to be used for measurements, due to the inherent limited resolution of a monochromator and the interference of the weaker collision-induced Rayleighwing spectrum. The difficulty associated with the grating monochromator is, however, alleviated in the interferometric experiment. Since not only is the instrumental resolution significantly increased, but also the Rayleigh wing appears as a flat background due to the overlapping of adjacent orders of the interferogram and
TABLE I. Rayleigh relaxation times $\left(\tau_{\text {Ray }}\right)$ and viscosity $(\eta)$ at various concentrations in the pyridine-water system.

\begin{tabular}{ccccc}
\hline \hline $\mathrm{Vol} \%$ & $\tau_{\text {Ray }}(\mathrm{ps})$ & $\tau_{\text {Ray }} / \eta(\mathrm{ps} / \mathrm{cP})$ & $\eta(\mathrm{cP})$ & $\eta / T(\mathrm{cP} / \mathrm{K})$ \\
\hline A. $20^{\circ} \mathrm{C}$ & & & & \\
10 & 11.2 & 8.75 & 1.28 & 0.00437 \\
33 & 16.1 & 8.59 & 1.88 & 0.00641 \\
44 & 16.1 & 7.21 & 2.24 & 0.00764 \\
55 & 15.5 & 6.25 & 2.48 & 0.00846 \\
77 & 12.0 & 5.02 & 2.39 & 0.00815 \\
83 & 10.3 & 5.00 & 2.06 & 0.00702 \\
90 & 9.3 & 6.08 & 1.53 & 0.00522 \\
95 & 7.7 & 6.41 & 1.20 & 0.00410 \\
100 & 4.3 & 4.40 & 0.98 & 0.00336 \\
& & & & \\
19 & 27.3 & 9.04 & 3.02 & 0.0111 \\
30 & 30.4 & 7.94 & 3.83 & 0.0140 \\
44 & 29.6 & 6.22 & 4.76 & 0.0174 \\
55 & 28.0 & 5.28 & 5.30 & 0.0194 \\
77 & 19.0 & 4.24 & 4.48 & 0.0164 \\
83 & 15.0 & 4.08 & 3.68 & 0.0135 \\
100 & 6.0 & 4.29 & 1.40 & 0.0051 \\
\hline \hline
\end{tabular}

does not interfere with the narrow spectral feature associated with molecular reorientation. For this reason, we believe that the values for $\tau_{\mathrm{Ray}}$ reported in Ref. 19 are subject to significant error.

As mentioned above, in general $\tau_{R_{2}}$ contains the pair correlation effect. $\tau_{\mathrm{R} a y}$ is related to $\tau_{s}$ through the relationship derived by Keyes and Kivelson ${ }^{1}$

$$
\tau_{\mathrm{Ray}}=\tau_{s}(1+f n) /(1+g n),
$$

where $n$ is the number density of scatteres and $f$ and $g$ are the static and dynamic OPC factors, respectively. Experimentally one finds that $g$ is much smaller than $f$ so that Eq. (3) can be approximately written as $\tau_{\mathrm{Ray}}$ $\approx \tau_{s}(1+f n)$.

Since $\tau_{s}$ (and $\tau_{\mathrm{R}_{\mathrm{ay}}}$ ) is a function of the solution viscosity, unambiguous $\tau_{s}$ and OPC values can only be obtained from the concentration dependent study, provided that the parameter $\kappa$ in Eq. (1) is independent of viscosity and temperature. In the case when the solution has a different shear viscosity from that of the neat liquid, the validity of Eq. (1), with $\kappa$ being temperature and viscosity independent, implies that $\tau_{\mathrm{Ra}}$ for the pure liquid can be obtained from the solution data by scaling the solution viscosity to that of the pure liquid. Using this technique, we have obtained recently in the pyridine$\mathrm{CCl}_{4}$ system the values of $\tau_{s}$ for pyridine at various temperatures. ${ }^{16}$ The OPC factor can also be determined by using this technique. In the pryidine $-\mathrm{CCl}_{4}$ study we have found that the OPC factor is negligible in neat pyridine.

Shown in Fig. 3 is the plot of Rayleigh relaxation times for different concentrations of pyridine in water as a 


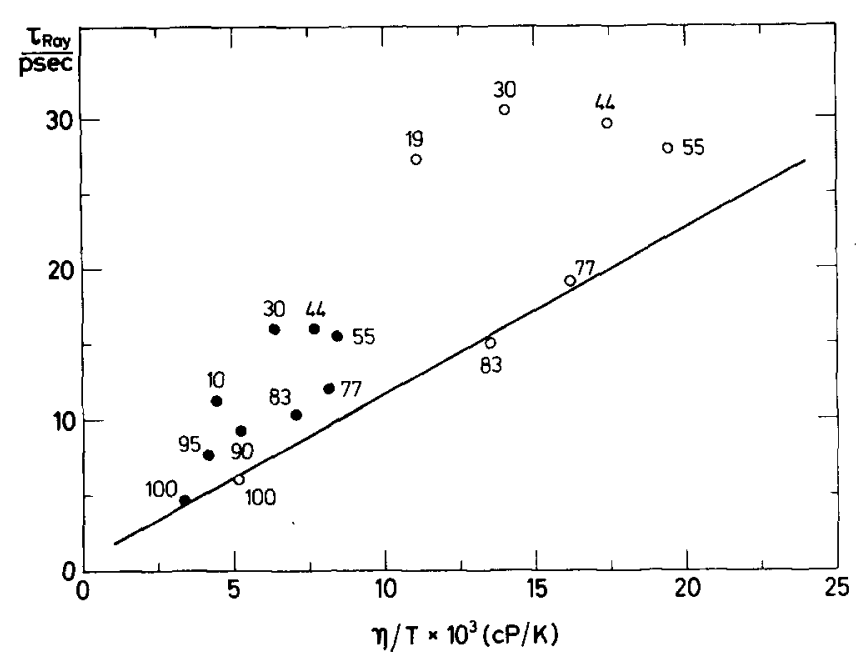

FIG. 3. Rayleigh relaxation time $\left(\tau_{\text {Ray }}\right)$ for different solutions of pyridine in water versus $\eta / T$ at $20^{\circ} \mathrm{C}$ (solid circles) and at $0^{\circ} \mathrm{C}$ (empty circles). Numbers indicate volume percent of pyridine and the line is the least squares fit to the pure pyridine points (only two points are shown in the diagram).

function of $\eta / T$ at $20^{\circ}$ and $0^{\circ} \mathrm{C}$. The solid circles are data obtained at $20^{\circ} \mathrm{C}$ and empty circles are those at $0^{\circ} \mathrm{C}$. The numeral indicates the volume percent of pyridine and the line represents the $\tau_{\mathrm{Ray}}$ versus $\eta / T$ result of pure pyridine (for which only two points are shown in the figure). Figure 3 shows clearly that the StokesEinstein-Debye (SED) equation with a concentration and temperature independent $k$ does not hold in the pyridine-water system. At high pyridine concentration (above $60 \mathrm{vol} \%$ ) the $\tau_{\mathrm{Ray}}$ values increases nonlinearly with increasing $\eta / T$; however, below this concentration. the $\tau_{\mathrm{Ray}}$ value remains more or less independent of $\eta / T$. Thus, except for the concentration at about $66 \mathrm{vol} \%$, $\tau_{\mathrm{Ray}}$ in the pyridine-water solution appears to be a double valued function of $\eta / T$, with the low pyridine solution having a greater $\tau_{\mathrm{R} \text { ay }}$ value. Significant departure from the classical SED equation (with a temperature and concentration independent $\kappa$ ) occurs in the low concentration. It is thus evident that the reorientational process associated with $\tau_{\mathrm{R} a \mathrm{y}}$ in the pyridine-water sys tem is not governed by the hydrodynamic effect alone; the nonhydrodynamic effect due to a specific intermolecular interaction between solute and solvent molecules plays an important role in affecting the $\tau_{\mathrm{R} 2 \mathrm{y}}$ values. In other words, the parameter $\kappa$ present in Eq. (1), which is related to the extent of the translational-rotation coupling of the reorienting molecules depends on the concentration and temperature of the pyridine-water solution.

Before proceeding further with the discussion of the nonhydrodynamic effect, a few words must be said about the static orientational pair correlation (OPC) factor. As pointed out above, our previous studies of the pyridine- $\mathrm{CCl}_{4}{ }^{16}$ and the pyridine-cyclohexane ${ }^{17}$ systems have shown that the OPC factor of neat pyridine liquid is negligible. Addition of a small amount of water may introduce short-range orientational order of pyridine through hydrogen-bonding interaction between one water and two pyridine molecules. This effect may enhance the OPC effect. Thus, the departure from the SED behavior of $\tau_{\mathrm{Ra}}$ with respect to $\eta / T$ at high pyridine concentration, shown in Fig, 3, may in part be due to the induced OPC effect as resulting from the hydrogen bond formation; however, it is difficult to separate the OPC effect from other mechanisms in this system because hydrogen bonding also induces other effects. Nevertheless, further addition of water to the solution will increase the average distance between pyridine molecules, and this will decrease the OPC effect. As is shown clearly in Fig. 3, great departure from the classical SED equation occurs at low pyridine concentration; thus, it is not likely that the OPC factor is responsible for the anomalous nonhydrodynamic behavior in the variation of $\tau_{\mathrm{Ray}}$ with respect to $\eta / T$. Instead, the departure from the classical SED equation for $\tau_{\mathrm{Ray}}$ is more likely due to the specific hydrogen-bonding interaction between pyridine and water. Such a type of interaction is expected to render the coupling parameter $\kappa$ strongly dependent on temperature, concentration, and viscosity.

The extent of deviation of $\tau_{R \text { ay }}$ from the classical SED equation can be shown by plotting the $\tau_{\mathrm{Ray}} / \eta$ data as a function of pyridine concentration at a fixed $T$, as shown in Fig. 4 for the cases of $T=0^{\circ} \mathrm{C}$ and $20^{\circ} \mathrm{C}$. Over the entire concentration range, two maxima at $x=0.05$ (about equal to 30 vol $\%$ pyridine) and at $x=0.8$ (about equal to $95 \mathrm{vol} \%$ ) are clearly shown. The maximum at low concentration can be understood as due to the incorporation of pyridine molecules in the water structure by the formation of the pyridine-water hydrogen bond. In the presence of the hydrogen bonding interaction, the reorientational dynamics of the pyridine molecule is altered drastically. The alteration causes not only the increase in the effective hydrodynamic volume but also the increase in the "stickness." The ultimate stickness value corresponds, of course, to $\kappa=1$. It should also be emphasized that the formation of the pyridine-water hydrogen bond also perturbs the molecular electronic distribution of the pyridine molecule. As

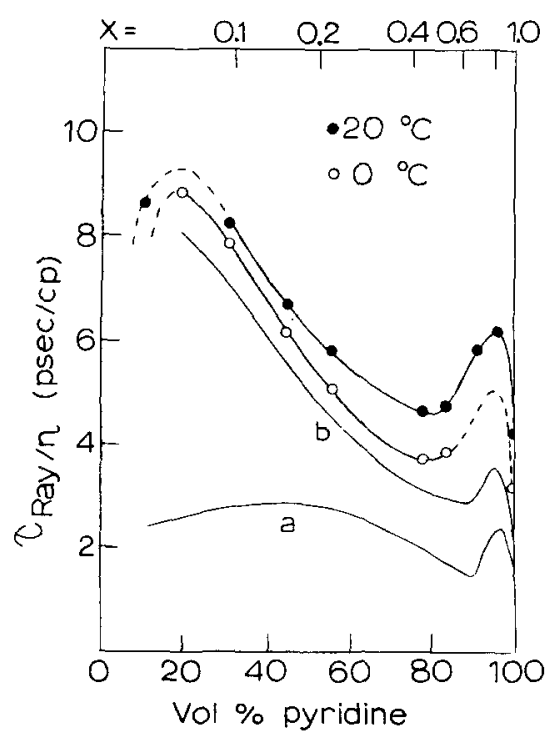

FIG. 4. $\tau_{\text {Ray }} / \eta$ plotted as a function of pyridine concentration for two temperatures $\left(0\right.$ and $\left.20^{\circ} \mathrm{C}\right)$. Corresponding plots from NMR relaxation data are included for comparison (see the text). 
a result, light scattering from the pyridine-water solution cannot be interpreted as being due to scattering from a system of uncorrelated symmetric tops. Nevertheless, considering the fact that the reorientational motion of pyridine about the $c$ axis perpendicular to the molecular plane requires a complete breaking of the hydrogen bond, this mode of reorientation is energetically less favorable when the pyridine concentration is low because of extensive hydrogen bonding. The reorientation about the $C_{2}(a)$ axis in the molecule does not require the hydrogen bond breaking and is expected to occur more readily. The reorientational motion about the $a$ axis is expected to be less temperature dependent. Unless only a small angle torsional oscillation occurs, requiring only the deformation of the $H$ bond, the reorientation about the $b$ axis (perpendicular to both $a$ and $c$ axes) also requires breaking of the $\mathrm{H}$ bond. Therefore, in the presence of water, the pyridine reorienta tional motion is anisotropic, as has been emphasized by Kintzinger ${ }^{25}$ in his NMR study of the aqueous solution of pyridine. The depolarized Rayleigh spectrum of pyridine in the presence of water is thus expected to consist of at least three Lorentzians; however, our attempts to fit the experimentally observed spectra (see Fig. 2) with more than one Lorentzian have not been successful, thus suggesting that the three types of reorientational motion probably do not differ too widely in the time scale, and depolarized Rayleigh spectroscopy is probably not sensitive enough to resolve the different types of reorientation processes. In view of this limitation, the depolarized Rayleigh scattering spectrum as shown in Fig. 2 reflects an average reorientation rate about the different axes.

As seen in Fig. 4, increasing the pyridine concentration beyond $20 \%(x=0.05)$ causes the $\tau_{\mathrm{Ray}} / \eta$ value to decrease with increasing concentration. In the concentration region between 20 and $80 \mathrm{vol} \%$, the $\tau_{\mathrm{R} \text { g }} / \eta$ value decreases steadily with increasing pyridine concentration. The decrease is possibly due to the presence of free pyridine molecules which reorient faster than the $\mathrm{H}$ bonded ones. Thus, in this region, $\tau_{\mathrm{Ray}}$ corresponds to an average of the reorientation times of the hydrogenbonded and the free pyridine molecules. As the concentration increases above $80 \%$, the $\tau_{\text {Ray }} / \eta$ value shows an increase with increasing the pyridine concentration, reaching a maximum at $x=0.8$ (or $95 \mathrm{vol} \%$ ). Beyond this concentration, $\tau_{\mathrm{Ray}} / \eta$ decreases with increasing pyridine concentration.

Included in Fig. 4 are also two curves labeled $a$ and $b$, obtained from Kintzinger's NMR results. ${ }^{25}$ Curves $a$ and $b$ indicate reorientation about the $a$ axis (the $C_{2}$ axis) and the $b$ axis (perpendicular to the $C_{2}$ axis in the molecular plane), respectively. While the NMR result is for $T$ $=25^{\circ} \mathrm{C}$ and are consistently smaller than the present $\tau_{\mathrm{R} \text { av }} / \eta$ values, the maximum in the $b$ curve occurs approximately in the same position as that in the $\tau_{\text {Rag }} / \eta$ data, corresponding to $x=0.8$. The maximum in curve $a$ occurs at higher concentration. Since the NMR experiment measures only the single particle reorientation time $\tau_{s}$, the maximum in the $\tau_{\mathrm{Ray}} / \eta$ curves cannot be interpreted as due to the OPC effect. It is possible that at high pyridine concentration the specific solute- solvent interaction favors the formation of hydrogen bonds involving one water and two pyridine molecules. Formation of such a complex increases significantly the effective volume for reorientation, and results in a large increase in the $\tau_{\mathrm{ray}}$ value. Since at high pyridine concentration molecules are also present, the maximum in $\tau_{\mathrm{Ray}} / \eta$ does not occur exactly at $x=0.66$, corresponding to $1: 2$ pyridine $-\mathrm{H}_{2} \mathrm{O}$ complex, despite the fact that the maximum is broad, covering the $x=0.66-0.8$ region.

As seen in Fig. 4, the $\tau_{\mathrm{R} \text { ay }} / \eta$ curve at $20^{\circ} \mathrm{C}$ lies above that at $0^{\circ} \mathrm{C}$, due to the fact that the viscosity of the pyridine solution at $0^{\circ} \mathrm{C}$ is conside rably greater than that at $20^{\circ} \mathrm{C}$ (see Fig. 1 and Table I). In view of this result, one notes that the difference between the depolarized Rayleigh scattering result and the NMR data is quite large. Although in Ref. 25 Kintzinger has analyzed the NMR data of the pyridine-water solution in terms of a completely anisotropic rotational diffusion tensor, his result cannot be verified in the Raman scattering experiment. ${ }^{19}$ The difficulty in the NMR study of the molecular reorientation process lies in the accurate determination of the electric field gradient at the relaxing nucleus and of the anisotropy in the nuclear quadrupole moment. In fact, even in the pure pyridine liquid, the depolarized Rayleigh scattering result already differs from the NMR result by almost a factor of $2 .^{16}$ Since there are numerous assumptions required in determining the molecular reorientation rate using NMR, we believe that the value that we have obtained in light scattering has a greater accuracy. Finally, it should be mentioned that Kintzinger also reported the reorientational relaxation time data about the $c$ axis. ${ }^{24}$ In this case, no maximum occurs at the high pyridine concentration region. At low pyridine concentrations $(x \simeq 0.1)$ the reorientational rate about the $c$ axis is about equal to that about the $b$ axis. Therefore, it is reasonable to argue that at low concentration the depolarized Rayleigh spectrum results from anisotropic reorientation about three axes, and at high concentration the reorientation about the $c$ axis contributes only negligibly to the spectral density. At low pyridine concentration, practically all pyridine molecules are involved in hydrogen bonding with water and because of the hydrogen-bonded structure which causes the reorientation of the pyridine molecules to become hindered, one expects to observe a long reorientational relaxation time. This is in agreement with the data shown in Fig. 3. According to Fig. 1, at $0^{\circ} \mathrm{C}$ the viscosity of the $20 \%$ solution is about equal to that of the $85 \%$ solution; nevertheless, the $\tau_{\mathrm{Ray}}$ value of the $20 \%$ solution is nearly twice as long as that of the $80 \%$ solution. Such a significant departure from the SED equation for $\tau_{\mathrm{R} \text { a }}$ of the low pyridine solution is associated with the nonhydrodynamic effect due to the extensive hydrogen-bond formation of the pyridine molecule with water. The activation energy for the reorientation of the pyridine molecule in aqueous solution can be obtained by measuring $\tau_{\mathrm{R} \text { r }}$ as a function of temperature, keeping the pyridine concentration fixed. The Arrhenius temperature dependence of $\tau_{\mathrm{Ra}}$, given by the equation

$$
\tau_{\mathrm{R} a \mathrm{y}}=\tau_{\mathrm{R} \mathbf{y}}^{0} \exp \left(E_{\mathrm{a}} / R T\right),
$$

is shown in Fig. 5 for a $77 \%$ solution of pyridine in 
water. At this concentration the activation energy $E_{a}$ as determined from the slope of the $\ln \tau_{\mathrm{Ray}}$ versus $1 / T$ plot is equal to $4.29 \mathrm{kcal} / \mathrm{mole}$.

For solutions other than 77 and $100 \%$, the temperature dependent measurements were not carried out in detail. We only made measurements at two temperatures and then assumed the Arrhenius equation to deduce the activation energy. The activation energy as deduced from the $\ln \tau_{\text {Ray }}$ versus $1 / T$ plot is shown as a function of pyridine concentration in Fig. 6. The activation energy deduced for NMR data ${ }^{27}$ is also included for comparison. As mentioned previously, the $\tau_{\mathrm{Ray}}$ data for the pyridinewater solution corresponds both to an average of reorientational relaxation times about the three principal axes and to an average value of bonded and free pyridine molecules; thus, it is rather surprising to note that the equation with only one activation energy as given by $\mathrm{Eq}$. (4) can fit so well the temperature dependence of the $\tau_{\text {Ray }}$ data of the pyridine-water solution for all concentrations. This result thus suggests that either only one type of the reorientational motion dominates the depolarized Rayleigh scattering or the activation energies are similar in all types of reorientational motions which contribute to the depolarized Rayleigh scattering spectral density of the pyridine-water solution. In the case of pure pyridine, only the reorientation of the molecular plane contributes to depolarized Rayleigh scattering. ${ }^{16}$ The activation energy for $\tau_{\mathrm{R} \text { a }}$ for pure pyridine is equal to $2.59 \mathrm{kcal} / \mathrm{mole}$, which is considerably smaller than the $E_{a}$ value for the aqueous solution when the hydrogenbonding interaction plays a significant role. At low pyridine concentration, practically all pyridine molecules are hydrogen bonded, and the activation energy should reflect the hydrogen bonding energy, considering the necessity of hydrogen-bond breaking for the reorientation of the pyridine molecules. The pyridine molecular reorientation in this concentration region is structure controlled. At $20 \%$ concentration, $E_{a}$ is equal to 4.7

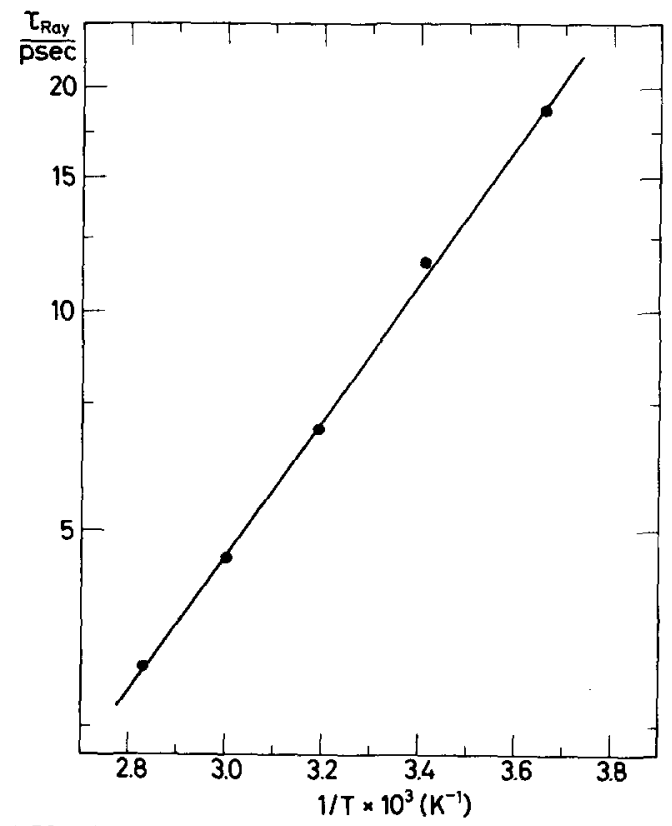

FIG. 5. Arrhenius plot of Rayleigh relaxation time versus inverse temperature for a $77 \%$ solution of pyridine in water.

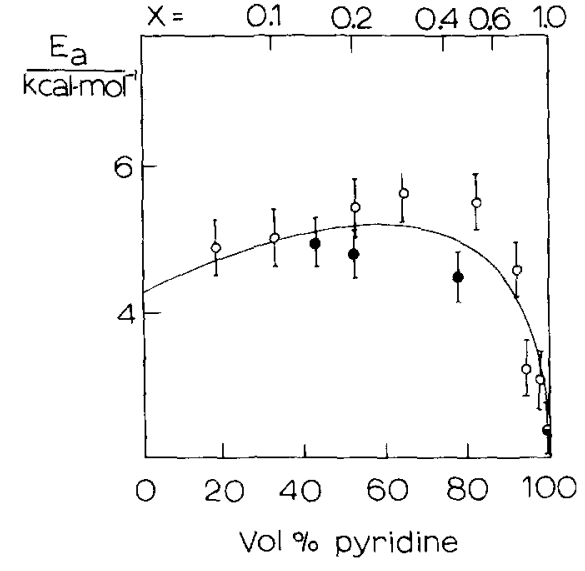

FIG. 6. Activation energy of Rayleigh relaxation time as a function of concentration (solid circles) and activation energy deduced from NMR data (empty circles, Ref. 27).

$\mathrm{kcal} / \mathrm{mole}$, which corresponds well to the value of the $\mathrm{N} \cdots \mathrm{H}-\mathrm{O}$ hydrogen bonding energy. ${ }^{26}$

On the other hand, in the concentration region $x>0.4$ (corresponding to about $70 \mathrm{vol} \%$ pyridine), the activation barrier to the reorientation decreases. This is understood as due to the breakdown of the water hydrogen-bonded network structure which results in the formation of $1: 1$ and $2: 1$ pyridine-water complexes. The $\tau_{R \text { ay }}$ data in this concentration range will therefore become less structure controlled. The increase in $\tau_{\text {Ray }}$ at high pyridine concentration is thus due to the increase in the effective hydrodynamic radius of the scattering particle.

\section{SUMMARY AND CONCLUSION}

We have studied the depolarized Rayleigh spectra of aqueous solutions of pyridine as a function of temperature and concentration. We have found that the depolarized Rayleigh relaxation time is affected strongly by the specific interaction due to the formation of hydrogen bonds between the lone pair of electrons on the nitrogen atom of the pyridine molecule and the hydrogen atoms of the water. In this system, the Rayleigh relaxation time, which reflects the molecular reorientation process, has a complex concentration and viscosity dependence. The classical Stokes-Einstein-Debye equation for molecular reorientation breaks down completely. At low pyridine concentration, the nonhydrodynamic effect associated with the formation of molecular complex dominates the molecular reorientation process. The role of the water network structure plays a significant role in affecting the reorientation of pyridine molecules. The $\tau_{\mathrm{R} \text { ay }}$ datum plotted as a function of $\eta / T$ is a double-valued function. At a given $\eta / T$, the low pyridine concentration solution has a longer $\tau_{\mathrm{R} \text { ay }}$ value.

At a fixed temperature, the plot of $\tau_{\mathrm{R} \text { ay }} / \eta$ versus pyridine concentration shows maxima at 30 and 95 vol $\%$ concentrations. The low concentration maximum is due to the incorporation of pyridine molecules in the water network structure and the high concentration maximum is associated with the formation of $1: 1$ and $2: 1$ pyridine-water complexes.

The Arrhenius-type temperature dependence has been 
found to correlate well the $r_{\mathrm{R} \text { g }}$ data for pyridine at all concentrations. The activation energy for molecular reorientation has been determined as a function of pyridine concentration. At low concentration, the activation energy is closely associated with the hydrogen bonding energy. As the concentration of pyridine is increased beyond $70 \mathrm{vol} \%$, the activation energy decreases with increasing pyriding concentration. Thus, we have shown that the nonhydrodynamic interaction plays an important role in the reorientational process of pyridine molecules in aqueous solutions is not determined by the macroscopic sheat viscosity of the solution.

\section{ACKNOWLEDGMENTS}

We thank the NATO Scientific Division and the NSF (grant number DMR 77-03707) for providing the financial support. One of the authors (P. -A. L.) wishes to thank the Danish Natural Science Research Council for a travel grant to Salt Lake City, where the experimental work was carried out.

${ }^{1}$ T. Keyes and D. Kivelson, J. Chem. Phys. 56, 1057 (1972).

${ }^{2}$ C. K. Cheung, D. R. Jones, and C. H. Wang, J. Chem. Phys. 64, 3567 (1976).

${ }^{3}$ T. D. Gierke and W. H. Flygare, J. Chem. Phys. 61, 2231 (1974).

${ }^{4}$ Y. Higashigaki, S. L. Whittenburg, and C. H. Wang, J. Chem. Phys. 69, 3297 (1978).

${ }^{5}$ D. Kivelson, M. G. Kivelson, and L. Oppenheim, J. Chem. Phys. 62, 1810 (1970).

${ }^{6} \mathrm{~J}$. Hwang, W. Z. Plachy, and D. Kivelson, J. Chem. Phys. 58, $1753(1973)$.
${ }^{7}$ D. Hoel and D. Kivelson, J. Chem. Phys. 62, 1323 (1975).

${ }^{8}$ P. Debye, Polar Molecules (Dover, New York, 1974).

${ }^{9}$ F. Perrin, J. Phys. Radium 5, 497 (1934).

${ }^{10}$ D. R. Bauer, J. I. Brauman, and R. Pecora, J. Am. Chem. Soc. $96,6840(1974)$ and references therein.

${ }^{11} \mathrm{~S}$. L. Whittenburg and C. H. Wang, J. Chem. Phys. BB, 4255 (1977) and references therein.

${ }^{12}$ R. R. Vold, S. W. Sparks, and R. L. Vold, J. Magn. Reson. 30, 497 (1978).

${ }^{13} \mathrm{C}$. $-\mathrm{M}$. Hu and R. Zwanzig, J. Chem. Phys. 60, 4354 (1974).

${ }^{14}$ M. D. Magee, Faraday Trans. II 929 (1974).

${ }^{15} \mathrm{C}$. H. Wang, J. Chem. Phys. 70, 3796 (1979).

${ }^{16}$ P. - A. Lund, S. L. Whittenburg, C. H. Wang, and D. H. Christensen, Mol, Phys. 37, 749 (1979).

${ }^{17}$ S. L. Whittenburg, D. R. Jones, D. H. Christensen, and C. H. Wang, J. Chem. Phys. 70, 2035 (1979).

${ }^{18}$ See, for example, H. Tokahashi, K. Mamula, and E. K. Plyler, J. Mol. Spectrosc. 21, 217 (1966).

${ }^{19} \mathrm{~W}$. Schindler and H. A. Posch, Chem. Phys. (to be published).

${ }^{20} \mathrm{~S}$. L. Whittenburg and C. H. Wang (unpublished results).

${ }^{21}$ D. H. Christensen, P. -A. Lund, S. L. Whittenburg, and C. H. Wang, Proc. Int. Conf. Raman Spectrosc., VIth, Bangalor, India, 1977 (1978).

${ }^{22} \mathrm{~J}$. Timmerans, The Physico-Chemical Constants of Binary Systems (Interscience, New York, 1959); J. Chem. Soc. London 93, 538 (1908).

${ }^{23}$ C. A. Long, F. S. Murtin, and E. I. Thomas, Trans. Faraday Soc. 58, 12 (1963); B. Bak, L. Hansen, and J. RastrupAnderson, J. Chem. Phys. 22, 2013 (1954).

${ }^{24}$ See, for example, D. R. Jones, C. H. Wang, D. H. Christensen, and P. -A. Lund, Chem. Phys. Lett. 38, 557 (1976).

${ }^{25} \mathrm{~J}$. P. Kintzinger, Mol. Phys. 30, 673 (1975).

${ }^{26} \mathrm{G}$. Pimentel and A. McClellan, The Hydrogen Bond (Freeman, San Francisco, 1960).

${ }^{27}$ E. V. Goldammer and H. G. Hertz, J. Phys. Chem. 74, 3734 (1970). 